\title{
Model predictive control of VSC-HVDC transmission system for power supply to passive networks
}

\author{
Pengfei Zhao ${ }^{1}$, Mingxing Guo ${ }^{1}$, Xiaojun Tang ${ }^{2}$, Yingpei Liu ${ }^{3}$, Qidi Huo ${ }^{2}$, Aili Pang ${ }^{1}$, Mengyao Zhang ${ }^{1}$, Sizhuo Lv $^{2}$ \\ ${ }^{1}$ State grid Shanghai electric power company economic and technological research institute, Xuhui District, Shanghai, 200233, China \\ ${ }^{2}$ China Electric Power Research Institute, Haidian District, Beijing, 100192, China \\ ${ }^{3}$ North China Electric Power University, Baoding, Hebei Province, 071003, China
}

\begin{abstract}
In the traditional double closed-loop control strategy for VSC-HVDC transmission system that supply power to passive networks, the control structure is complex, the PI parameters are more difficult, the tuning is slow, and the response speed is slow. Rectifier-side direct power control based on model prediction and direct $\mathrm{AC}$ voltage control strategy on inverter side are proposed. Based on the discrete mathematical model of the converter, the system output under all switching function combinations is calculated by the ergodic method, and the switching function that minimizes the objective function is selected to act on the inverter. The utility model has the advantages of simple structure, no complicated PI parameter setting, fast dynamic response, high voltage quality electric energy to the passive network, good steady state performance and dynamic performance. The simulation results verify the feasibility and effectiveness of the proposed control strategy.
\end{abstract}

\section{Introduction}

With the Development of Controllable Turn-off Power Electronic Devices and PWM, high-voltage direct current transmission systems based on voltage source converters (VSC-HVDC) are receiving increasing attention. Compared with the traditional HVDC transmission system, VSC-HVDC has less harmonic content and can independently control active and reactive power [1]. It has broad application prospects in the fields of new energy generation and grid connection, power supply to weak AC grid or passive network [2-6].

The VSC-HVDC control strategy for powering passive networks is currently a research hotspot. Reference [7] is based on the discretization mathematical model of the converter and uses the digital PI controller to design the power outer loop of the rectifier side, the current inner loop controller and the inverter side fixed AC voltage controller. The proposed controller was simulated and verified under various working conditions. Reference [8] proposes a double closed-loop control strategy for the inner loop voltage outer loop of the inverter-side current. Since the response speed of the voltage outer loop is much lower than the response speed of the current inner loop, the voltage quality is easily affected when the load side is disturbed. In the Reference [9], based on the analysis of the mathematical model of VSC converter, the control strategy of VSC converter is designed. The inner loop adopts the deadbeat control to realize the error-free tracking of the control variables. In [10], the direct power control of the rectifier side of the
VSC-HVDC system and the direct AC voltage control strategy of the inverter side are proposed based on the model predictive control. The selection method of the weight coefficient is described in detail, and the simulation example is used to explain and verify in detail. Overcome the subjectivity and blindness of weight coefficient selection.

In this paper, the discrete mathematical model of the VSC-HVDC rectification side and the inverter side for power supply to the passive network is established. The rectifier side direct power control and the inverter side direct AC voltage control based on model prediction are proposed. The method has simple control structure and overcomes the shortcomings of the traditional double closed-loop control strategy, such as multiple PI parameters and difficulty in setting. The dynamic response speed is fast, and can provide higher voltage quality electric energy to the passive network, and has good steady state performance and dynamic performance. Finally, based on MATLAB/Simulink platform, a VSCHVDC system for powering passive networks is built, and the proposed control strategy is verified.

\section{Mathematical model of VSC-HVDC}

The structure of the VSC-HVDC system that supplies power to the passive network is shown in Figure 1. Both the rectifier and the inverter consist of a three-phase twolevel converter. In Figure $1, Z_{s}$ is the equivalent resistance of the AC system; the inductance of the $L_{1}$ and $L_{2}$ converters; $R_{1}$ and $R_{2}$ are the total equivalent resistance of the commutating reactor and the three-phase converter; $\boldsymbol{u}_{1}$

$\overline{{ }^{*} \text { Corresponding author's e-mail: liuyingpei_123@126.com }}$ 
is the AC grid voltage vector; $\boldsymbol{u}_{1}{ }^{\prime}$ is the outlet voltage vector of the rectifier side converter station; $\boldsymbol{i}_{1}$ is the rectifier side current vector; $\boldsymbol{u}_{2}$ is the load voltage vector; $\boldsymbol{u}_{2}{ }^{\prime}$ is the inverter side converter AC voltage vector; $\boldsymbol{i}_{2}$ is the inverter side current vector; $\boldsymbol{i}_{\mathrm{L}}$ is the load Current vector; $u_{1 d c}$ and $u_{2 d c}$ are DC voltages on the rectification side and inverter side respectively; $C$ is a DC capacitor; $C_{2}$ and $L_{2}$ form an LC low-pass filter for filtering out higher harmonics, while $C_{2}$ provides voltage support to the passive network.

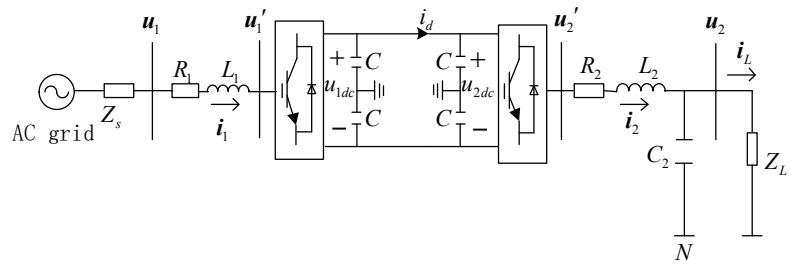

Figure 1. Structure Diagram of Two-level VSC-HVDC System Supplying Power to Passive Load

\subsection{Rectification side mathematical model}

When the AC system is balanced, according to the system structure, electric quantity reference direction and Kirchhoff's law in Figure 1, a mathematical model of the rectification side in a three-phase stationary coordinate system can be obtained:

$$
L_{1} \frac{d i_{1 k}}{d t}=u_{1 k}-R_{1} i_{1 k}-u_{1 d c}\left(\mathrm{t}_{1 k}-\frac{1}{3} \sum_{n=a, b, c} t_{1 n}\right)
$$

In equation (1), $k=a, b, c ; t_{1 k}$ is the switching function of the $k$ th phase bridge arm of the rectifier. The switching function reflects the switching state of each phase bridge arm of the rectifier, and can be defined as follows: $t_{1 k}=\left\{\begin{array}{lc}0 & \text { The upper arm closes and the lower arm opens } \\ 1 & \text { The upper arm leads through and the lower arm closes }\end{array}\right.$

According to the variation formula of the $a b c$ coordinate system to the $\alpha \beta$ coordinate system:

$$
\left[\begin{array}{l}
\alpha \\
\beta
\end{array}\right]=\frac{2}{3}\left[\begin{array}{ccc}
1 & -\frac{1}{2} & -\frac{1}{2} \\
0 & \frac{\sqrt{3}}{2} & -\frac{\sqrt{3}}{2}
\end{array}\right]\left[\begin{array}{l}
a \\
b \\
c
\end{array}\right]
$$

The expression of formula (1) in the $\alpha \beta$ coordinate system is as follows:

$$
L_{1} \frac{d \boldsymbol{i}_{1}}{d t}=\boldsymbol{u}_{1}-R_{1} \boldsymbol{i}_{1}-u_{1 d c} \boldsymbol{t}_{1}
$$

In the formula (4), $\boldsymbol{i}_{1}=\left[\begin{array}{ll}i_{1 \alpha} & i_{1 \beta}\end{array}\right]^{\mathrm{T}} . i_{1 \alpha}, i_{1 \beta}$ are the components of the rectifying side alternating current on the $\alpha$ and $\beta$ axes; $\boldsymbol{u}_{1}=\left[\begin{array}{cc}u_{1 \alpha} & u_{1 \beta}\end{array}\right]^{\mathrm{T}}, u_{1 \alpha}, u_{1 \beta}$ are the grid voltages on the $\alpha$ and $\beta$ axes. The upper component; $\boldsymbol{t}_{1}=\left[t_{1 \alpha}\right.$. $\left.t_{1 \beta}\right]^{\mathrm{T}}, t_{1 \alpha}, t_{1 \beta}$ are the components of the rectifier switching function on the $\alpha$ and $\beta$ axes.

Discretization of the formula (4) using the first-order forward difference method yields:

$$
\boldsymbol{i}_{1}(k+1)=\left(1-\frac{R_{1} T_{s}}{L_{1}}\right) \boldsymbol{i}_{1}(k)+\frac{T_{s}}{L_{1}}\left[\boldsymbol{u}_{1}(k)-u_{1 d c}(k) \boldsymbol{t}_{1}(k)\right]
$$

In equation (5), $T_{s}$ is the system sampling period.
For three-phase two-level converters, the three-phase bridge arm has eight possible combinations of switch states:

$$
\left[\begin{array}{l}
t_{1 a}(k) \\
t_{1 b}(k) \\
t_{1 c}(k)
\end{array}\right] \in\left\{\left[\begin{array}{l}
0 \\
0 \\
0
\end{array}\right],\left[\begin{array}{l}
0 \\
0 \\
1
\end{array}\right],\left[\begin{array}{l}
0 \\
1 \\
0
\end{array}\right],\left[\begin{array}{l}
0 \\
1 \\
1
\end{array}\right],\left[\begin{array}{l}
1 \\
0 \\
0
\end{array}\right],\left[\begin{array}{l}
1 \\
0 \\
1
\end{array}\right],\left[\begin{array}{l}
1 \\
1 \\
0
\end{array}\right],\left[\begin{array}{l}
1 \\
1 \\
1
\end{array}\right]\right\}
$$

According to equation (3), the commutation station switching state function is converted to the $\alpha \beta$ coordinate system, and $\boldsymbol{t}_{1}(k)$ has seven possible states, which are:

$$
\left[\begin{array}{l}
t_{1 \alpha}(k) \\
t_{1 \beta}(k)
\end{array}\right] \in\left\{\left[\begin{array}{l}
0 \\
0
\end{array}\right],\left[\begin{array}{c}
-\frac{1}{3} \\
-\frac{\sqrt{3}}{3}
\end{array}\right],\left[\begin{array}{c}
-\frac{1}{3} \\
\frac{\sqrt{3}}{3}
\end{array}\right],\left[\begin{array}{c}
-\frac{2}{3} \\
0
\end{array}\right],\left[\begin{array}{l}
\frac{2}{3} \\
0
\end{array}\right],\left[\begin{array}{c}
\frac{1}{3} \\
-\frac{\sqrt{3}}{3}
\end{array}\right],\left[\begin{array}{c}
\frac{1}{3} \\
\frac{\sqrt{3}}{3}
\end{array}\right]\right\}
$$

\subsection{Inverter side mathematical model}

When the AC system is balanced, according to the system structure, electric quantity reference direction and Kirchhoff's law in Figure 1, the mathematical model of the inverter side in the three-phase stationary coordinate system can be obtained:

$$
\left\{\begin{array}{l}
L_{2} \frac{d i_{2 k}}{d t}=-u_{2 k}-R_{2} i_{2 k}+u_{2 d c}\left(\mathrm{t}_{2 k}-\frac{1}{3} \sum_{n=a, b, c} t_{2 n}\right) \\
C_{2} \frac{d u_{2 k}}{d t}=i_{2 k}-i_{L k}
\end{array}\right.
$$

In equation (8), $k=a, b, c ; t_{2 k}$ is the switching function of the $k$ th phase bridge arm of the inverter. Its definition is as follows:

$t_{2 k}=\left\{\begin{array}{lc}0 & \text { The upper arm closes and the lower arm opens } \\ 1 & \text { The upper arm leads through and the lower arm closes }\end{array}\right.$

By performing the coordinate transformation of equation (8), the mathematical model of the inverter side in the $\alpha \beta$ coordinate system can be obtained as follows:

$$
\left\{\begin{array}{l}
L_{2} \frac{d \boldsymbol{i}_{2}}{d t}=-\boldsymbol{u}_{2}-R_{2} \boldsymbol{i}_{2}+u_{2 d c} \boldsymbol{t}_{2} \\
C_{2} \frac{d \boldsymbol{u}_{2}}{d t}=\boldsymbol{i}_{2}-\boldsymbol{i}_{L}
\end{array}\right.
$$

In the formula (10), $\boldsymbol{i}_{2}=\left[\begin{array}{ll}i_{2 \alpha} & i_{2 \beta}\end{array}\right]^{\mathrm{T}}, i_{2 \alpha}, i_{2 \beta}$ are components of the alternating current on the $\alpha$ and $\beta$ axes of the inverter side; $\boldsymbol{u}_{2}=\left[\begin{array}{ll}u_{2 \alpha} & u_{2 \beta}\end{array}\right]^{\mathrm{T}}, u_{2 \alpha}, u_{2 \beta}$ are the components of the load voltage on the $\alpha$ and $\beta$ axes; $\boldsymbol{t}_{2}=\left[\begin{array}{ll}t_{2 \alpha} & t_{2 \beta}\end{array}\right]^{\mathrm{T}}, t_{2 \alpha}, t_{2 \beta}$ are the components of the inverter switching function on the $\alpha$ and $\beta$ axes; $i_{L}=\left[\begin{array}{ll}i_{L \alpha} & i_{L \beta}\end{array}\right]^{\mathrm{T}}, i_{L \alpha}, i_{L \beta}$ are the components of the load current on the $\alpha$ and $\beta$ axes.

Rewrite equation (10) into the form of a state equation:

$$
\left\{\begin{array}{l}
\frac{d \boldsymbol{X}}{d t}=\boldsymbol{A} \boldsymbol{X}+\boldsymbol{A}_{1} u_{2 d c} \boldsymbol{t}_{2}+\boldsymbol{A}_{2} \boldsymbol{i}_{L} \\
\boldsymbol{Y}=\boldsymbol{C} \boldsymbol{X}
\end{array}\right.
$$

In equation (11), $\boldsymbol{X}$ is the state variable, $\boldsymbol{X}=\left[\begin{array}{lll}i_{2 \alpha} & i_{2 \beta} & u_{2 \alpha}\end{array}\right.$ $\left.u_{2 \beta}\right]^{\mathrm{T}}$; the input is $u_{2 d c} \boldsymbol{t}_{2}=\left[\begin{array}{ll}u_{2 d c} t_{2 \alpha} & u_{2 d c} t_{2 \beta}\end{array}\right]^{\mathrm{T}}$; the disturbance is $\boldsymbol{i}_{L}=\left[\begin{array}{ll}i_{L \alpha} & i_{L \beta}\end{array}\right]^{\mathrm{T}}$; the output $\boldsymbol{Y}=\left[\begin{array}{ll}u_{2 \alpha} & u_{2 \beta}\end{array}\right]^{\mathrm{T}}$.

$$
\boldsymbol{A}=\left[\begin{array}{cccc}
-R_{2} / L_{2} & 0 & -1 / L_{2} & 0 \\
0 & -R_{2} / L_{2} & 0 & -1 / L_{2} \\
1 / C_{2} & 0 & 0 & 0 \\
0 & 1 / C_{2} & 0 & 0
\end{array}\right] ; \quad \boldsymbol{A}_{1}=\left[\begin{array}{cc}
1 / L_{2} & 0 \\
0 & 1 / L_{2} \\
0 & 0 \\
0 & 0
\end{array}\right] ;
$$




$$
\boldsymbol{A}_{2}=\left[\begin{array}{cc}
0 & 0 \\
0 & 0 \\
-1 / C_{2} & 0 \\
0 & -1 / C_{2}
\end{array}\right] ; \quad \boldsymbol{C}=\left[\begin{array}{llll}
0 & 0 & 1 & 0 \\
0 & 0 & 0 & 1
\end{array}\right]
$$

Discretization of equation (11) yields a discretized state equation as:

$$
\left\{\begin{array}{l}
\boldsymbol{X}(k+1)=\boldsymbol{B} \boldsymbol{X}(k)+\boldsymbol{B}_{1} u_{2 d c}(k) \boldsymbol{t}_{2}(k)+B_{2} \boldsymbol{i}_{L}(k) \\
\boldsymbol{Y}(k+1)=\boldsymbol{C} \boldsymbol{X}(k+1)
\end{array}\right.
$$

where

$$
\begin{gathered}
\boldsymbol{B}=e^{\boldsymbol{A} T_{s}} \\
\boldsymbol{B}_{1}=\int_{0}^{T_{s}} e^{\boldsymbol{A} \tau} \boldsymbol{A}_{1} d \tau=\left(e^{\boldsymbol{A} T_{s}}-\boldsymbol{E}\right) \boldsymbol{A}^{-1} \boldsymbol{A}_{1} \\
\left.\boldsymbol{B}_{2}=\int_{0}^{T_{s}} e^{\boldsymbol{A} \tau} \boldsymbol{A}_{2} d \tau=\left(e^{\boldsymbol{A} T_{s}}-\boldsymbol{E}\right) \boldsymbol{A}^{-1} \boldsymbol{A}_{2}\right)
\end{gathered}
$$

In the formulas (14) to (15), $\boldsymbol{E}$ is a $4 \times 4$ unit matrix.

Similar to $t_{1}(k), t_{2}(k)$ has seven possible states in the $\alpha \beta$ coordinate system.

\section{Controller design of VSC-HVDC system}

When the VSC-HVDC system that supplies power to the passive network is in normal operation, the rectifier side adopts constant DC voltage and constant reactive power control to maintain the stability of the DC system; the inverter side uses a constant $\mathrm{AC}$ voltage to provide a stable AC voltage to the load to ensure normal operation of the load.

\subsection{Design of Rectifier Side Controller}

According to the instantaneous power theory, the predicted values of active power and reactive power from the grid side to the rectifier side converter station are:

$$
\begin{aligned}
P_{1}(k+1)= & R\left[\left[\boldsymbol{u}_{1}(k+1) \dot{i_{1}}(k+1)\right]=R \mathrm{e}\left[\left[u_{1 \alpha}(k+1)+j u_{1 \beta}(k+1)\right]\left[i_{1 \alpha}(k+1)-j i_{1 \beta}(k+1)\right]\right]\right. \\
& =u_{1 \alpha}(k+1) i_{1 \alpha}(k+1)+u_{1 \beta}(k+1) i_{1 \beta}(k+1) \\
Q_{1}(k+1) & =\operatorname{Im}\left[\boldsymbol{u}_{1}(k+1) \dot{\boldsymbol{i}}_{1}(k+1)\right]=\operatorname{Im}\left[\left[u_{1 \alpha}(k+1)+j u_{1 \beta}(k+1)\right]\left[i_{1 \alpha}(k+1)-j i_{l_{\beta}}(k+1)\right]\right] \\
& =u_{1 \beta}(k+1) i_{1 \alpha}(k+1)-u_{1 \alpha}(k+1) i_{1 \beta}(k+1)
\end{aligned}
$$

where $\boldsymbol{i}_{1}(k+1)$ is the current predicted value obtained by equation (5). Since the sampling frequency of the system is much higher than the grid frequency, $\boldsymbol{u}_{1}(k)=\boldsymbol{u}_{1}(k+1)$ can be considered.

The active power command value flowing to the rectification side can be obtained by equation (18):

$$
P_{1}^{*}=k_{p}\left(u_{1 d c}{ }^{*}-u_{1 d c}\right)+k_{i} \int\left(u_{1 d c}{ }^{*}-u_{1 d c}\right) d t+u_{1 d c} i_{d}
$$

In the equation (18), $u_{1 d c}{ }^{*}$ is the rectification side DC voltage command value.

In order to stabilize the active power and reactive power at the command value, the following objective function is constructed:

$$
g_{1}=\delta_{P 1}\left|P_{1}^{*}-P_{1}(k+1)\right|+\delta_{Q 1}\left|Q_{1}^{*}-Q_{1}(k+1)\right|
$$

In formula (19), $\delta_{p 1}$ and $\delta_{q 1}$ are weight coefficients.

The weight coefficients $\delta_{p 1}$ and $\delta_{q 1}$ in the objective function respectively indicate the proportion of the active power and the reactive power in the control strategy. The larger the weight coefficient is, the more important the corresponding control objectives are.

The control strategy of the rectifier side converter station is shown in Figure 2. The control strategy constructs a corresponding objective function according to the active power and the reactive power control target, finds a switch state that minimizes the objective function, and realizes direct control of power.

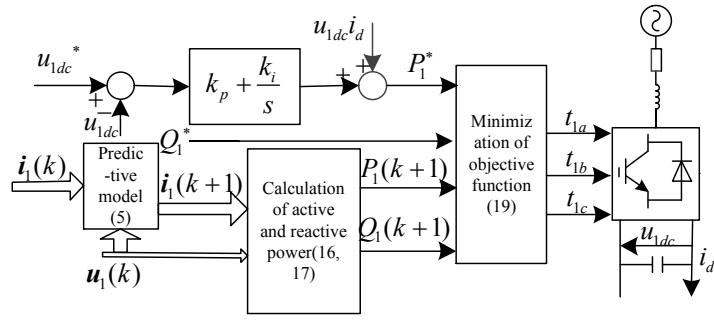

Figure 2. Rectification side control strategy

\subsection{Inverter side controller design}

The inverter side control objective is to maintain the load $\mathrm{AC}$ voltage stable and enable the load to operate at rated conditions. Therefore, first set the command value of the load AC voltage:

$$
\begin{aligned}
& u_{2 a}{ }^{*}(k+1)=u_{m}{ }^{*} \sin \left(2 \pi f^{*}(k+1) T_{s}\right) \\
& u_{2 b}{ }^{*}(k+1)=u_{m}{ }^{*} \sin \left(2 \pi f^{*}(k+1) T_{s}-120^{\circ}\right) \\
& u_{2 c}{ }^{*}(k+1)=u_{m}{ }^{*} \sin \left(2 \pi f^{*}(k+1) T_{s}+120^{\circ}\right)
\end{aligned}
$$

where $u_{m}{ }^{*}$ is the rated amplitude of the load AC voltage. $f^{*}$ is the rated frequency of the load, $f^{*}=50 \mathrm{~Hz}$.

Through the $\alpha \beta$ coordinate transformation, the command value of the load AC voltage in the $\alpha \beta$ coordinate system can be obtained:

$$
\left[\begin{array}{l}
u_{2 \alpha}{ }^{*}(k+1) \\
u_{2 \beta}{ }^{*}(k+1)
\end{array}\right]=\frac{2}{3}\left[\begin{array}{ccc}
1 & -\frac{1}{2} & -\frac{1}{2} \\
0 & \frac{\sqrt{3}}{2} & -\frac{\sqrt{3}}{2}
\end{array}\right]\left[\begin{array}{l}
u_{2 a}{ }^{*}(k+1) \\
u_{2 b}{ }^{*}(k+1) \\
u_{2 c}{ }^{*}(k+1)
\end{array}\right]
$$

In order for the load $\mathrm{AC}$ voltage to accurately track its given value, the following objective function is constructed:

$$
g_{2}=\left[\boldsymbol{u}_{2}^{*}(k+1)-\boldsymbol{u}_{2}(k+1)\right]^{2}=\left[u_{2 \alpha}{ }^{*}(k+1)-u_{2 \alpha}(k+1)\right]^{2}+\left[u_{2 \beta}{ }^{*}(k+1)-u_{2 \beta}(k+1)\right]^{2}
$$

$\boldsymbol{u}_{2}(k+1)$ is the voltage predicted value obtained by equation (12).

The inverter side control strategy is shown in Figure 3. The control strategy constructs a corresponding objective function according to the load AC voltage control target, finds a switch state that minimizes the objective function, and realizes direct control of the load AC voltage.

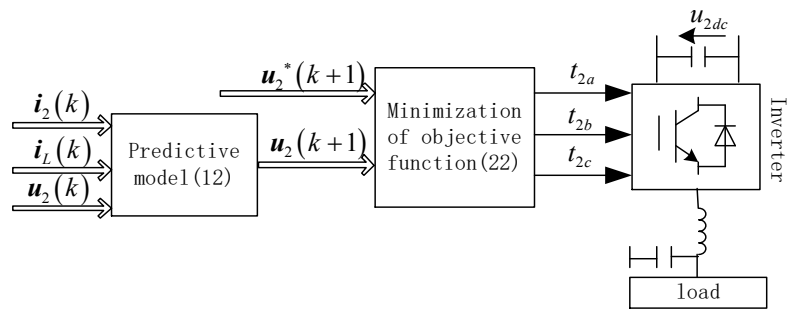

Figure 3. Inverter side control strategy 
The process of model predictive control includes the following seven steps:

(1) Measure the relevant electrical quantity and obtain the sampled value at the $k$ th time. The rectification side is $i_{1}(k), u_{1}(k), u_{1 d c}(k)$; the inverter side is $u_{2 d c}(k), i_{2}(k), u_{2}(k)$, $i_{L}(k)$.

(2) Calculate the constant coefficient. For example, $1-\frac{R_{1} T_{s}}{L_{1}}$ in equation (5) and the matrix $\boldsymbol{B}, \boldsymbol{B}_{1}, \boldsymbol{B}_{2}$ in the formula (12).

(3) The predicted value at the $k+1$ th time is calculated based on the prediction model, the sampled value at the $k$ th time, and the seven possible switch states.

(4) According to the objective function, the objective function values corresponding to the seven switch states are calculated.

(5) Compare the seven objective function values calculated above, and select the switching state that minimizes the objective function.

(6) Apply the optimal switch state combination to the inverter.

(7) Repeat steps (1) to (6) in the next control cycle.

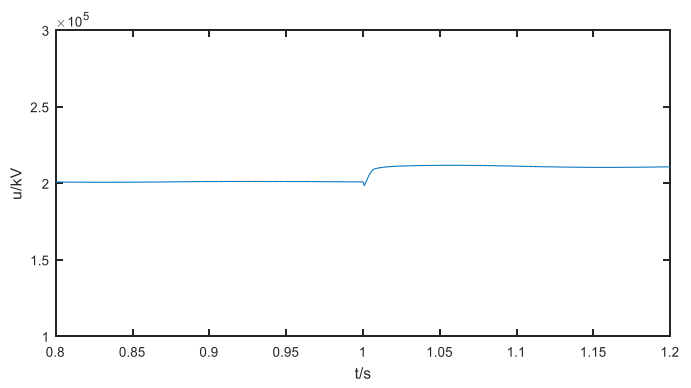

(a) Rectifier side DC voltage waveform

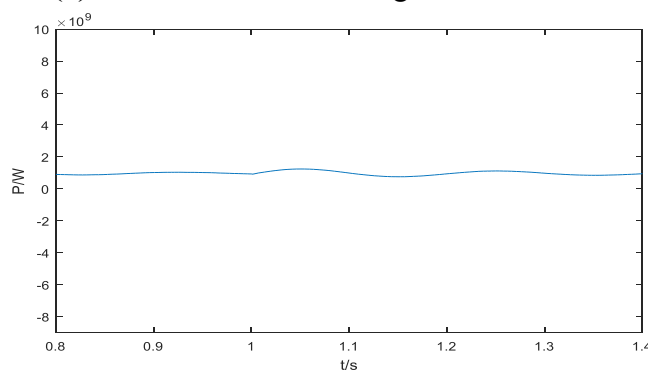

(c) Rectification side active power waveform

\section{Simulation analysis}

In order to verify the effectiveness of the proposed control strategy, the VSC-HVDC simulation model for powering the passive network shown in Figure 1 is built by MATLAB. Simulation parameters are shown in Table 1.

Table 1. System simulation parameters.

\begin{tabular}{ll}
\hline Parameter & $\begin{array}{l}\text { Numerical } \\
\text { value }\end{array}$ \\
\hline Rectification side grid line voltage $u_{1 a b} / \mathrm{kV}$ & 110 \\
Rectifier side DC voltage command value & 200 \\
$u_{1 d c}{ }^{*} / \mathrm{kV}$ & 9000 \\
$\mathrm{DC}$ capacitor $C / \mu \mathrm{F}$ & 110 \\
Load AC line voltage command value & \\
$u_{2 a b}{ }^{*} / \mathrm{kV}$ & 0.01 \\
Inverter side inductance $L_{2} / \mathrm{mH}$ & 850 \\
Inverter side capacitor $C_{2} / \mu \mathrm{F}$ & 5 \\
The sampling period $T_{s} / \mu \mathrm{s}$ & \\
\hline
\end{tabular}

\subsection{DC voltage mutation}

In the initial state, the rectified side DC voltage command value is $200 \mathrm{kV}$, the reactive power command value is 1000Mvar, and the inverter side load AC line voltage command value is $110 \mathrm{kV}$. In order to test the performance of the rectifier side controller designed in this paper, the rectifier side DC voltage command value is stepped from $200 \mathrm{kV}$ to $210 \mathrm{kV}$ at $1 \mathrm{~s}$. The simulation results are shown in Figure 4.

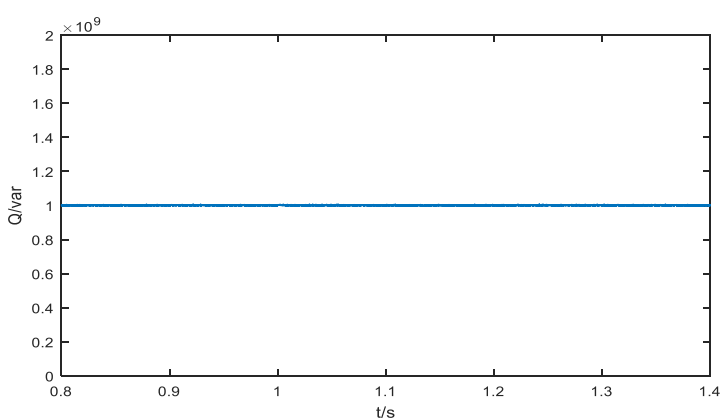

(b) Rectification side reactive power waveform

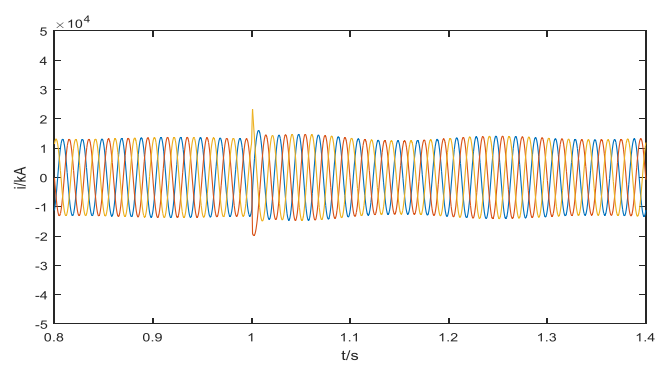

(d) Rectifier side alternating current

Figure 4. Rectifier side DC voltage abrupt simulation waveform

It can be seen from the simulation results that after $1 \mathrm{~s}$, after the DC voltage command value is stepped, the DC voltage reaches a new steady state value after about 0.01 $\mathrm{s}$, and the steady state error is 0 . When the DC voltage command value is stepped, the active power of the rectification side fluctuates, and the reactive power of the rectification side remains unchanged, and is always maintained at 1000 Mvar, realizing independent control of active and reactive power. The three-phase alternating current of the rectification side is completely symmetrical, and has good dynamic performance and waveform quality. It can be seen from the above analysis that the rectifier side controller performs well. 


\subsection{Load burst}

In the initial state, the rectification side DC voltage command value is $200 \mathrm{kV}$, the reactive power command value is $0 \mathrm{Mvar}$, and the inverter side load AC line voltage

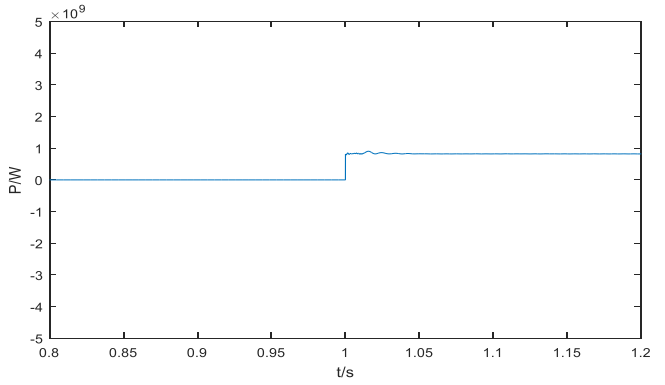

(a) Load active power waveform

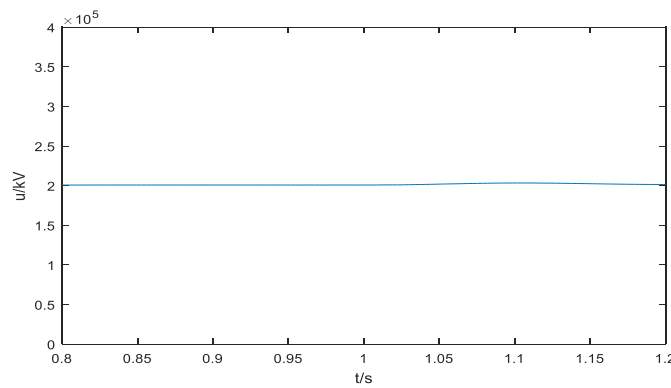

(c) DC voltage waveform command value is $110 \mathrm{kV}$, and the passive network is unloaded. In order to test the dynamic performance of the inverter side controller, a $15 \Omega$ linear load is input to the passive network at $1 \mathrm{~s}$. The simulation results are shown in Figure 5.

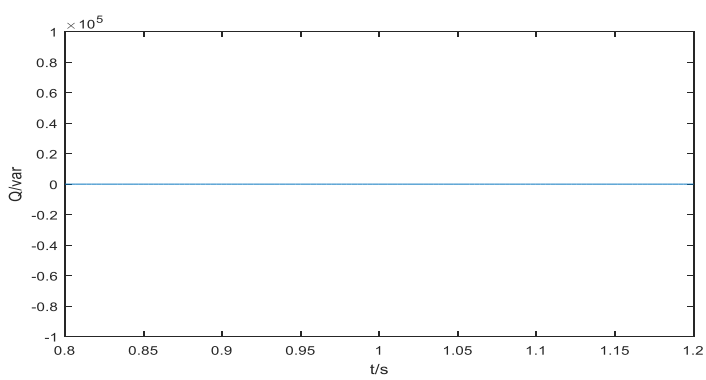

(b) Load reactive power waveform

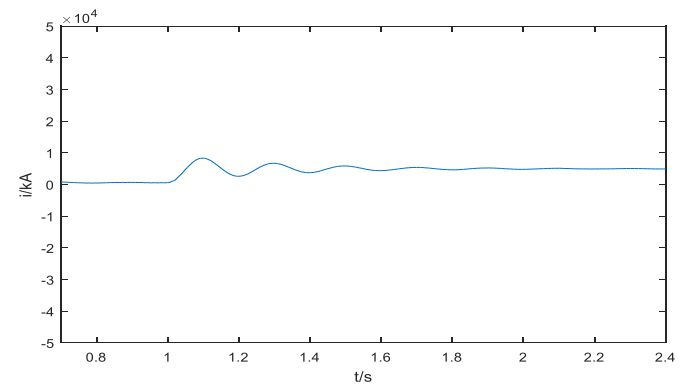

(d) DC current waveform

Figure 5. Load burst simulation waveform

It can be seen from the simulation results that the load is abrupt at $1 \mathrm{~s}$. The DC voltage is not affected by the inverter side, and is always maintained at $200 \mathrm{kV}$. After the load active power increases, the DC current increases from 0 to $5 \mathrm{kA}$. In addition, both the load voltage and the load current are three-phase symmetrical and the waveform quality is good. It can be seen from the above analysis that the dynamic performance of the inverter side controller is good.

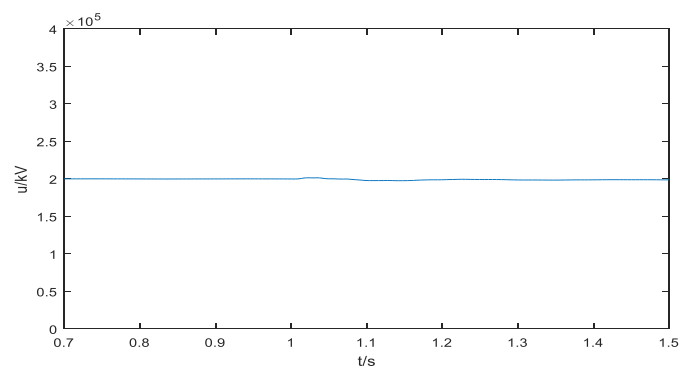

(a) Rectifier side DC voltage waveform

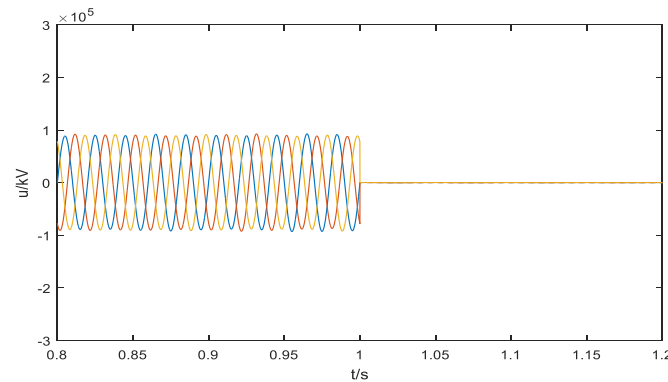

(c) Load AC voltage waveform

\subsection{Three-phase ground fault}

In the initial state, the rectified side DC voltage command value is $200 \mathrm{kV}$, the reactive power command value is OMvar, and the inverter side load $\mathrm{AC}$ line voltage command value is $110 \mathrm{kV}$. At $1 \mathrm{~s}$, a three-phase ground short circuit fault occurs on the inverter side. The simulation results are shown in Figure 7.

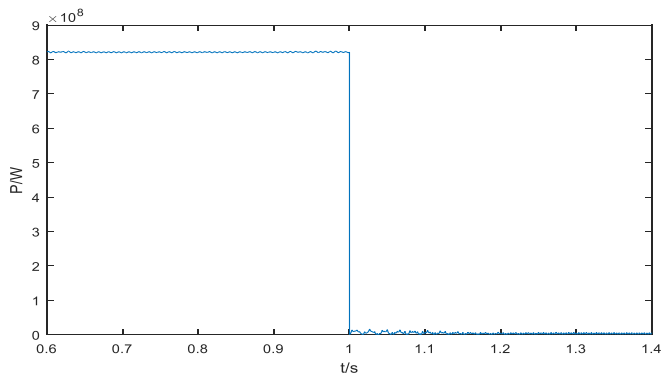

(b) Load active power waveform

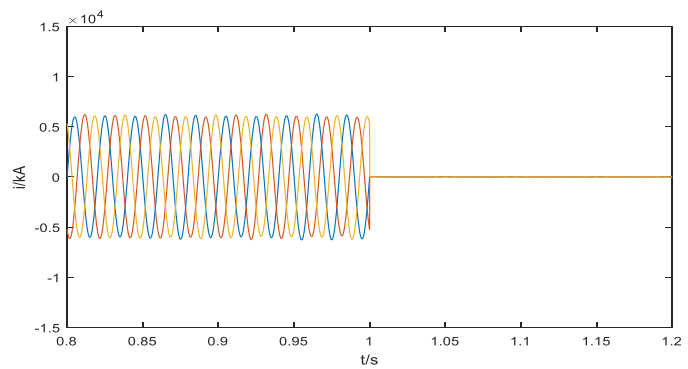

(d) Load AC current waveform

Figure 6. Inverter side three-phase grounding simulation waveform 
It can be seen from the simulation results that after the three-phase short-circuit fault occurs on the inverter side, the three-phase voltage on the inverter side becomes 0 , so the active power of the three-phase load current and the load consumption also becomes 0 . The DC voltage is hardly affected by the inverter side fault and is always maintained at $200 \mathrm{kV}$. It can be seen from the above analysis that the rectifier side controller performs well after the three-phase grounding occurs on the inverter side.

\section{Conclusions}

In this paper, the discrete mathematical model of the rectifier side and the inverter side of the VSC-HVDC system powered by the passive network are derived. Based on the discrete mathematical model, the rectifier side direct power control and the inverter side direct AC voltage control based on the model predictive control are designed. Simulation results verify the effectiveness of the proposed control strategy. The main conclusions are as follows:

(1) The method omits the current inner loop control, the control structure is simple, and no complicated PI parameter tuning is needed, which improves the system reliability.

(2) The dynamic response is fast, and it can provide higher voltage quality electric energy to the passive network, and has good steady state performance and dynamic performance.

\section{References}

1. Xu, D. G., Liu, Y. C., Wu, J. (2015) Overview of multi-terminal HVDC transmission control research. Journal of Electrical Engineering, 30(17): 1-12.

2. Zhang, L., Harnefors, L., Nee, H. P. (2011) Interconnection of Two Very Weak AC Systems by VSC-HVDC Links Using Power-Synchronization Control. IEEE Transactions on Power Systems, 26(1): 344-355.

3. Sun, X. Y., Tong, X. Q., Gao, X. (2014) Fault diagnosis method for IGBT valve in flexible DC transmission system. Journal of Electrical Engineering, 29(8): 235-241.

4. Jiang, G. Q., Li, Z. Y., Yang, H. X., et al. (2015) Summary of research on topology of flexible DC transmission system. Power System Protection and Control, 15: 145-153.

5. Wang, Z. H., Li, Y., Gao, K., et al. (2015) Research on additional damping control of VSC-HVDC with fault recovery signal. Power System Protection and Control, 43(21): 34-40.

6. Zhang, L., Harnefors, L., Nee, H. P. (2011) Modeling and Control of VSC-HVDC Links Connected to Island Systems. IEEE Transactions on Power Systems, 26(2): 783-793.

7. Yang, H., Zhang, N., Ye, M. J. (2012) VSC-HVDC discrete model for power supply to passive networks and its control strategy. Power System Protection and
Control, 40(4): 37-42.

8. Guan, M. Y., Xu, Z. (2013) Modeling and control of MMC DC transmission system for power supply to passive networks. Journal of Electrical Engineering, 28 (2): 255-263.

9. Wang, W., Shi, X., Meng, J., et al. (2013) Modeling and control of VSC-HVDC supplying passive network. Advanced Materials Research, 45(5): 213217.

10. Liang, Y. Y., Zhang, T., Liu, J. Z., et al. (2015) VSCHVDC model predictive control for power supply to passive networks. Journal of Electrical Engineering, 30(11): 78-89. 